\title{
Technical Overview on the Electron Backscattered Diffraction Sample Preparation
}

\author{
Dong-Ik Kim ${ }^{1, *}$, Byung-Kyu Kim ${ }^{1,2}$, Ju-Heon Kim ${ }^{1,2}$ \\ ${ }^{1}$ High Temperature Energy Materials Research Center, Korea Institute of Science and Technology, Seoul 02792, Korea \\ ${ }^{2}$ Department of Materials Science and Engineering, Seoul National University, Seoul 08826, Korea
}

*Correspondence to:

Kim DI,

Tel: +82-2-958-5432

Fax: +82-2-958-5379

E-mail: dongikkim@kist.re.kr

Received November 20, 2015

Revised December 3, 2015

Accepted December 3, 2015
A technical overview on the various sample preparation methods for electron backscattered diffraction (EBSD) analysis is carried out. The mechanical polishing with colloidal silica finish, electro-chemical polishing, dual layer coating and ion beam milling are introduced for the common sample preparation methods for EBSD observation and some issues that are frequently neglected by the common EBSD users but should be considered to get a reliable EBSD data are discussed. This overview would be especially helpful to the people who know what EBSD technique is but do not get a reliable EBSD data because of difficulties in sample preparation.

Key Words: Electron backscattered diffraction, Sample preparation, Mechanical polishing, Electro-chemical polishing, Ion milling

\section{INTRODUCTION}

Since Venables and Harland (1973) introduced electron back scattered diffraction (EBSD) technique to obtain the crystallographic information in scanning electron microscope (SEM), there have been huge progresses in hardware and software of EBSD systems. Automatic indexing of EBSD patterns using Hough transformation (Lassen, 1994), high resolution EBSD using field emission gun SEM (Humphreys, 1999), and phase identification using energy dispersive spectroscopy (EDS)-EBSD combination (Kim et al., 2013) are the examples of those meaningful advances in EBSD systems. These days, EBSD is not only a tool for obtaining the crystallographic information of the samples but also a commonly used microstructure observation tools because it can provide colorful images and statistical microstructural information such as grain size and grain boundary characteristics at the same time (Kim et al., 2004; Jung et al., 2015). Even though EBSD becomes a commonly installed attachment comparable to EDS that people select when they purchase a new SEM system, people still experience difficulties in getting a good EBSD patterns for various specimens and sometimes fail to get a reliable EBSD data, which mainly comes from the inadequate sample preparation. In this paper, we would like to overview commonly used EBSD sample preparation techniques and address the key issues that a new EBSD user should keep in mind to get a reliable EBSD result.

\section{GENERAL OVERVIEW OF EBSD SAMPLE PREPARATION}

The main reason why people experience difficulties in getting a good EBSD pattern is the interaction volume of EBSD pattern formation is too small. It is only 10 to $40 \mathrm{~nm}$ from the sample surface (Schwartz et al., 2010). To compose a diffraction pattern, there should be a periodic arrangement of atoms inside the interaction volume of incident electron beam and the specimen. In case that the sample surface is slightly deformed during the sample preparation or contaminated, we cannot secure an enough volume of sound atomic array

The authors acknowledge the support provided by the Technology Innovation Program (Metal SOFC Interconnect thick plate (15 t) and thin plate (250 $\mu \mathrm{m})$ VIM Production Technology (500 kg) Development) funded by the Ministry of Trade, Industry and Energy (MOTIE, Korea).

(a) This is an open-access article distributed under the terms of the Creative Commons Attribution Non-Commercial License (http://creativecommons.org/licenses/by-nc/4.0) which permits unrestricted noncommercial use, distribution, and reproduction in any medium, provided the original work is properly cited.

Copyrights @ 2015 by Korean Society of Microscopy 
Table 1. Typical electrolyte and electro chemical polishing condition for EBSD sample preparation

\begin{tabular}{llcc}
\hline Materials & \multicolumn{1}{c}{ Electrolyte } & Voltage $^{\text {Flow rate }}$ & \\
\hline Most metals & $5 \% \sim 10 \%$ perchloric+ethanol $\left(-30^{\circ} \mathrm{C}\right)$ & $25 \sim 30$ & $16 \sim 20$ \\
Stainless steels & $5 \% \sim 10 \%$ perchloric+ethanol (room temperature) & 6 & 16 \\
SUS 301 & 590 mL methanol+350 mL methyl propanol+60 mL perchlolic acid $\left(-30^{\circ} \mathrm{C}\right)$ & 18 & 20 \\
& Perchloric acid $(25 \%)+$ ethanol $(40 \%)+$ water $(30 \%)+2$-butoxyethanol $(5 \%)^{\dagger}$ & $11 \sim 14$ & 15 \\
\hline
\end{tabular}

*Flow rate indicates the value of Struers electro polisher LectroPol 5.

${ }^{\dagger}$ Known as A2 solution by Strueres.
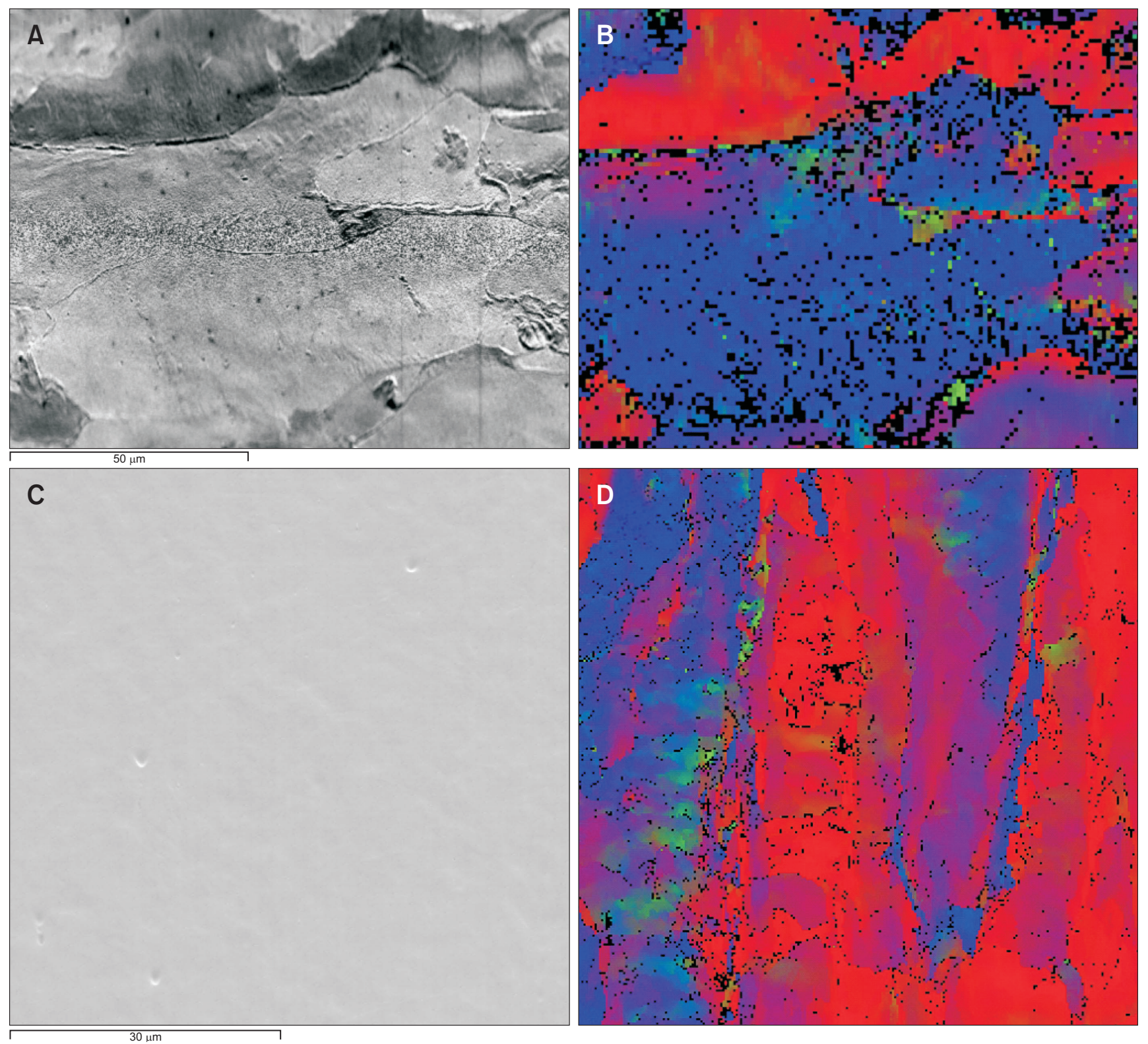

Fig. 1. A comparison of a room temperature and low temperature electro-chemical polishing in interstitial free steels scanning electron micrograph (A), normal direction orientation map acquired by the room temperature electro-chemical polishing (B), scanning electron micrograph (C), and normal direction orientation map acquired by electro-chemical polishing at $-30^{\circ} \mathrm{C}(\mathrm{D})$. 
to compose a diffraction pattern. If we compare it with the EDS analysis case, the interaction volume of EDS signal in typical analysis condition is around $1 \mu \mathrm{m}$ and the $\mathrm{X}$-ray signal comes from the each interacting atom independently. So we don't need to pay too much attention on the surface status for EDS analysis as we do for EBSD analysis. For EBSD analysis, to keep the interacting top surface layer residual strain free is essential for a good EBSD pattern.

A basic method to get the residual strain free surface is doing a final polishing with colloidal silica suspension that is a chemically reactive. It polishes the sample surface not only mechanically but also chemically and remains less residual strain on the sample surface. A common mistake that the new EBSD users often make is doing colloidal silica final polishing too long time. Colloidal silica polishing is also a kind of chemical treatment and there is an optimum time for the best result. In most cases 1 to 3 minutes polishing is recommended for the best EBSD patterns.

Another useful sample preparation method for EBSD in most metallic specimens are electro-chemical polishing. Because the EBSD analysis technique is an application using a SEM with shallow interaction volume, mounting of the specimens that is typically used for the specimen polishing is not recommended. Commonly using mounting resins are nonconductive and the metal sputter coating, which is typically used for the SEM observation, is also not applicable because a typical thickness of the coating layer is thicker than the theoretical EBSD pattern formation depth. When we prepare specimens with electro-chemical polishing, we don't need to worry about this conductivity issue because we can prepare the specimens without mounting. The second merit of the electro-chemical polishing is that a fine surface preparation is not required. Grinding up to 1,200 or 2,000 grid silicon carbide paper is enough for the surface preparation prior to the electro-chemical polishing. Another advantage of electro-chemical polishing is less than one minutes polishing is enough for each sample. From these characteristics, the electro-chemical polishing is mainly recommended for the repeated observation of metallic samples with similar compositions. Table 1 shows some examples of electrolyte and electro-chemical polishing condition combinations for several materials. For the most metals, perchloric acid and ethyl alcohol mixture can be applied. Electro-chemical polishing can be done at room temperature but it will show the better results when it is done at low temperature $\left(-30^{\circ} \mathrm{C}\right)$ with higher voltage. Fig. 1 shows the SEM micrographs and EBSD orientation maps of interstitial free steels prepared at room temperature and $-30^{\circ} \mathrm{C}$. When it is prepared at room temperature, slightly etched microstructure is acquired and it is easy to define a desired observing site in SEM. While when it is prepared at $-30^{\circ} \mathrm{C}$, the sample surface is too smooth and it is rather difficult to see the microstructure in secondary electron image mode. On the other hand it has advantage in the data acquisition rate. It is increasing from $84.3 \%$ to $95.5 \%$ in these specimens. Furthermore most EBSD suppliers provide forward scattered electron detectors in front of EBSD cameras and it provides the composition and orientation contrast images. So the disadvantage of low temperature sample preparation is negligible in most cases.

\section{KEY ISSUES OF EBSD SAMPLE PREPARATION}

In some cases, even though we pay much attention on the sample surface condition, we often fail to get a good EBSD patterns. A basic but the most important thing to keep in mind in EBSD sample preparation is understanding of
A

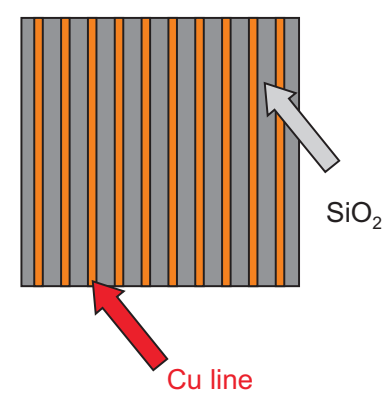

B

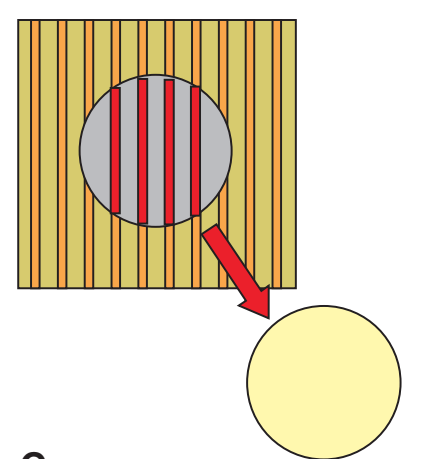

C

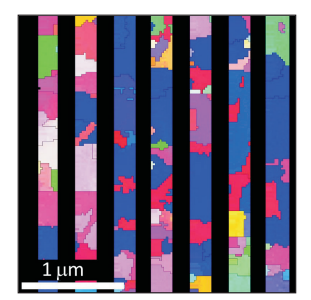

D

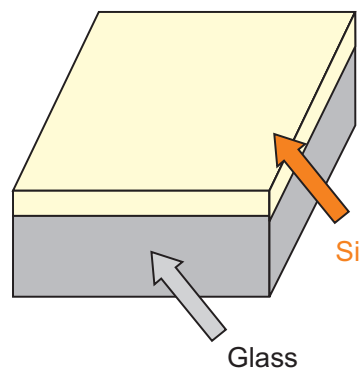

E

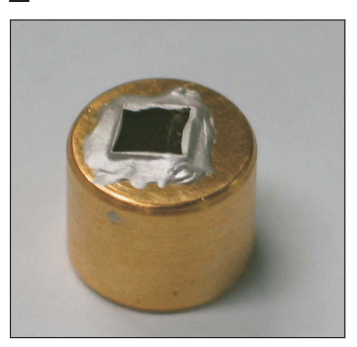

Fig. 2. (A) Schematic layout of Cu damascene line sample. (B) Dual layer coating for the better conductivity. (C) Normal direction orientation map of $\mathrm{Cu}$ damascene line sample. (D) Schematic layout of Si thin film for liquid crystal display (LCD) application. (E) Silver paste deposition for the electrical conduction. (F) Normal direction orientation map of poly Si sample. Scale bar $=5 \mu \mathrm{m}$ 
samples' structure. Fig. 2 shows a typical examples how the EBSD sample preparation should be changed according to the samples' structure. The fist example shown as the Fig. 2A-C is a $\mathrm{Cu}$ damascene line which the sample surface consists of two phases with different conductivity levels, $\mathrm{Cu}$ and silicon oxide. In this multi phases surface case, it is hard to prevent charging during the EBSD mapping and it often results in wavy orientation maps in spite that the damascene lines are straight (Muppidi et al., 2005). For this case, both of increasing overall conductivity of the sample and keeping the conductivity of whole sample area in a similar level are the critical issues to prevent the charging during the EBSD mapping. Kim et al. (2002) introduced dual layer coating to prevent this problem. They applied a thick gold coating first to increase overall conductivity with a mask to preserve the observing area from the thick coating which can deteriorate the EBSD pattern quality. But this gold coating added an additional phase on the sample surface and make the charging problem severer than prior to the gold coating. So a thin carbon coating of 10 $\mathrm{nm}$ thick was applied to make the whole sample surface keep similar conductivity level. Fig. 2C shows the results acquired by this dual layer coating and a straiht lines without wavy
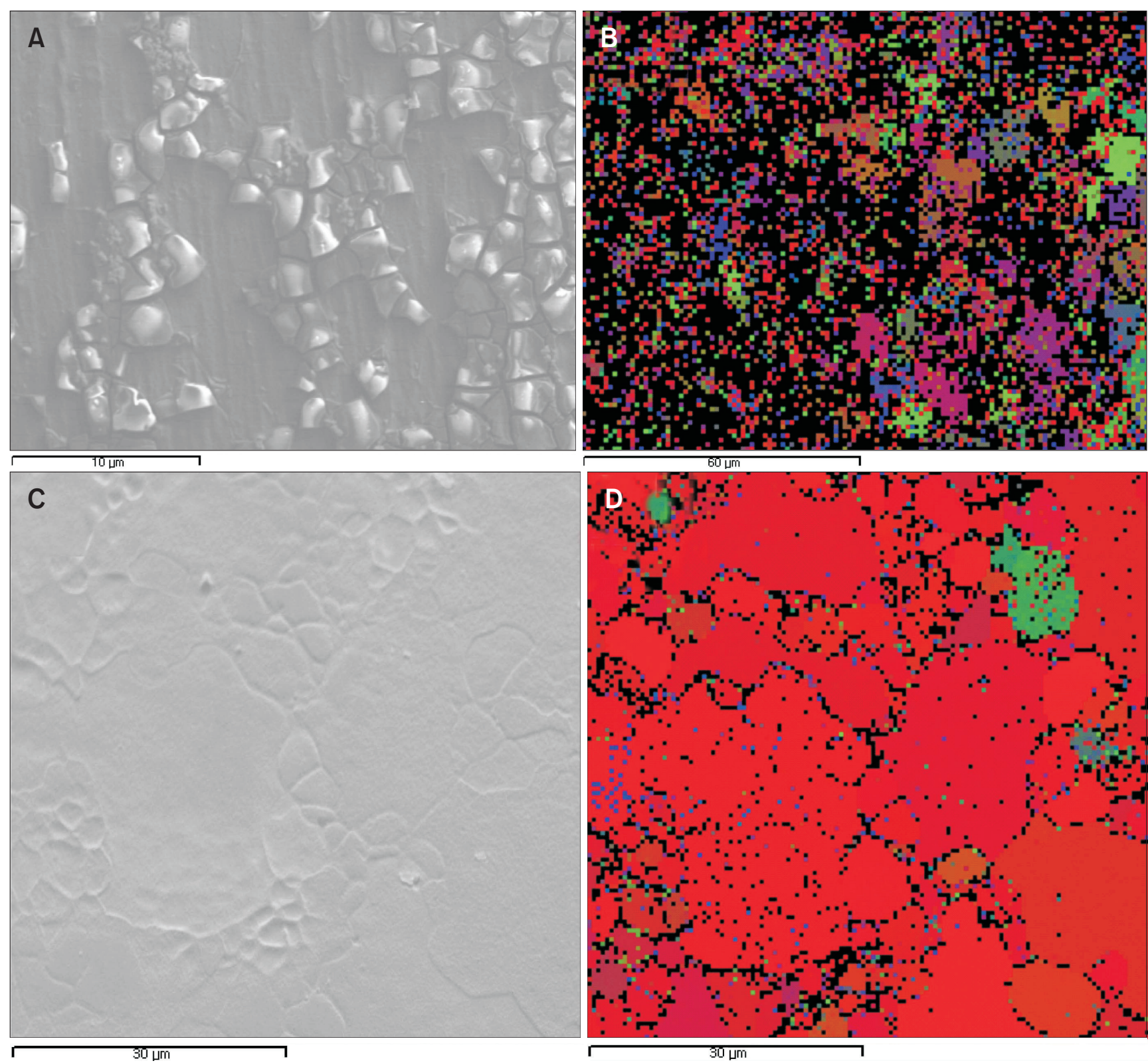

Fig. 3. Mg sample prepared by typical electro-chemical polishing scanning electron microscope (SEM) micrograph of the sample (A) and normal direction orientation map (B). Mg sample prepared by non-aqueous solutions SEM micrograph of the sample (C) and normal direction orientation map (D). 
curvature was acquired.

Fig. 2D-F show the EBSD mapping of Si thin film for the liquid crystal display (LCD) application. This Si thin film is normally deposited on glass and irradiated electrons for EBSD observation are accumulated on the Si layer and also causes charging during EBSD observation. Then simple connection of electron circuit from the sample surface to the metallic sample holder is enough to prevent this charging as shown in Fig. 2E. Fig. 2F shows that a reliable EBSD data can be acquired by this simple silver paste deposition. One more thing to be considered for the thin Si layer EBSD observation is that a silicon oxide layer can be easily formed on the sample surface of the thin film and deteriorate EBSD pattern quality seriously. To prevent this, cleaning of the sample in the $10 \%$ Hydrofluoric acid and 90\% distilled water mixture for 30 seconds before mounting on the sample holder with silver paste.

Another example of the importance of sample structure understanding is reactive metals like $\mathrm{Mg}$. $\mathrm{Mg}$ can be also prepared by electro-chemical polishing with the electrolyte of $5 \%$ to $10 \%$ perchloric and ethanol mixture. But it is so reactive and the surface can be easily oxidized as shown in Fig. 3A during the post sample treatment and the EBSD results are not reliable (Fig. 3B). So it is critical to use absolute Ethanol without water composition for the electrolyte and the cleaning agent as well. And the fast drying of the sample after electro-chemical polishing is also recommended. With this careful treatment, the surface condition of the sample and the EBSD data quality can be improved dramatically as shown in Fig. 3C and D. Mg samples can also be prepared by mechanical polishing. But in this case also, non-aqueous resin such as alcohol based and oil based ones should be used to
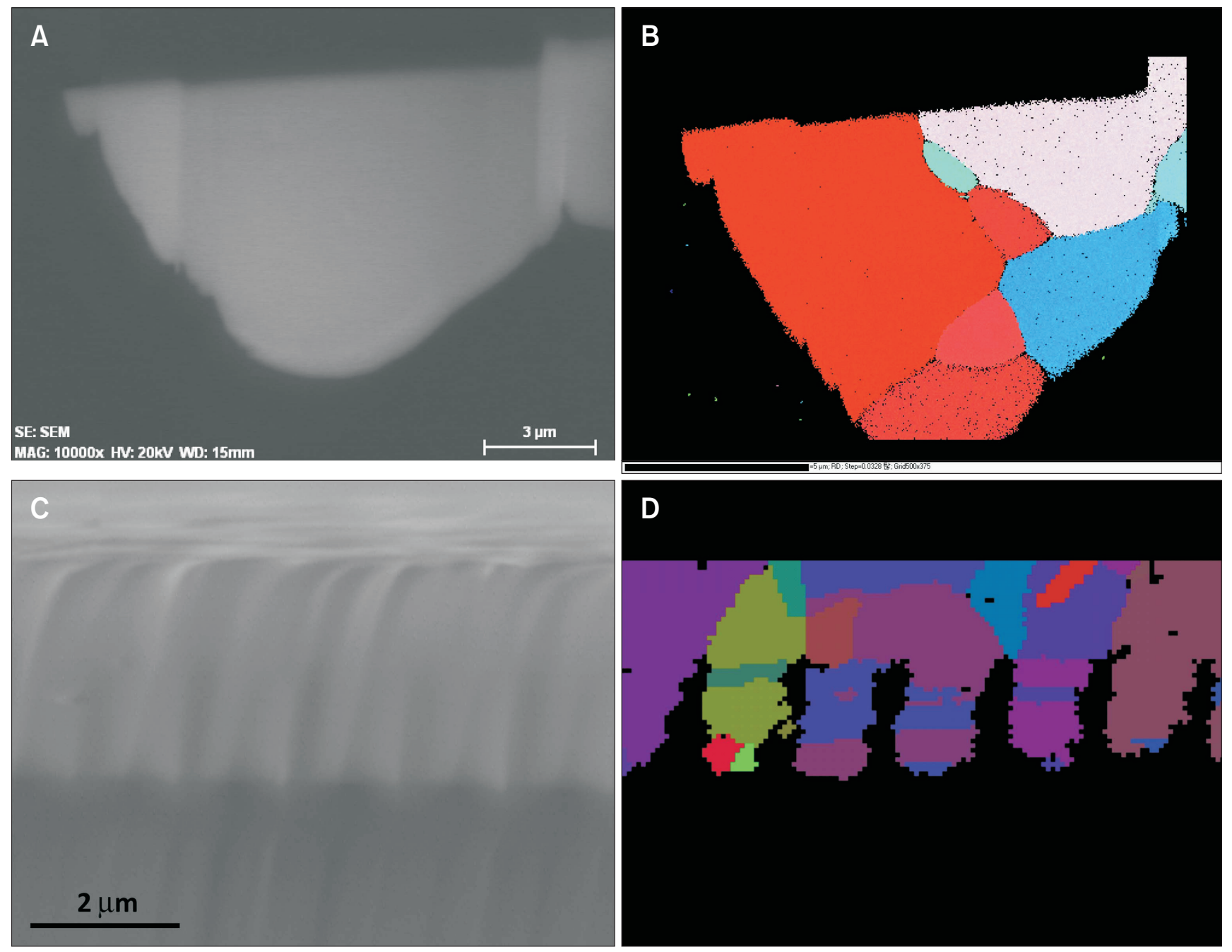

Fig. 4. Electron back scattered diffraction (EBSD) data acquired from indium zinc oxide transmission electron microscope sample prepared by focused ion beam (FIB) scanning electron microscope (SEM) micrograph (A), normal direction orientation map (B; scale bar=5 $\mu \mathrm{m}$ ) and EBSD data acquired from the cross sectional site of the $\mathrm{Cu}$ damascene line sample prepared by FIB cross section milling SEM micrograph (C), transverse direction orientation map (D). 
prevent the surface oxidation.

\section{SAMPLE PREPARATION WITH ION BEAM}

Another technique that becomes popular recently in EBSD sample preparation is ion beam milling. Several companies provide cross sectional ion beam milling machines or flat type ion millers. Some people use focused ion beam (FIB) machines for EBSD sample preparation also. It is commonly believed that an ion beam milling does not leave any residual strain on the sample surface, which is the main reason why it becomes so popular recently. However this hypothesis is sometimes correct but sometimes wrong. Normally ion beam milling is a technique to take off the molecules from the sample with ion beam irradiation and it affects the sample surface during the ion milling. Fig. 4 shows EBSD data acquired from indium zinc oxide transmission electron microscope sample (Fig. 4A and B) and cross sectional of a
$\mathrm{Cu}$ damascene line sample (Fig. 4C and D) prepared by FIB. Both samples show no clear EBSD pattern when they were prepared by $25 \mathrm{kV}$ Ga ion beam because the irradiated high energy Ga ions deteriorate the periodic atomic arrangement at the sample surface and make the diffracted pattern diffuse. With the additional fine milling with ion beam accelerated 5 $\mathrm{kV}$ or less voltage, the samples produce clear EBSD pattern and a reliable EBSD data can be acquired as shown in Fig. 4.

These ion beam damage is not serious in the cross sectional ion milling case. For the flat surface milling with planar ion miller, an ion beam damage during the milling is accumulated on the sample surface continuously. So we can define the optimum milling time to get a good EBSD pattern and it is normally less than 2 minutes at 5 to $6 \mathrm{kV}$ milling. But for the cross sectional milling case, people observe the site that is perpendicular to the incident ion beam. It means that the observing surface is protected by the ion beam shield and the damaged layer that was affected by the ion beam irradiation
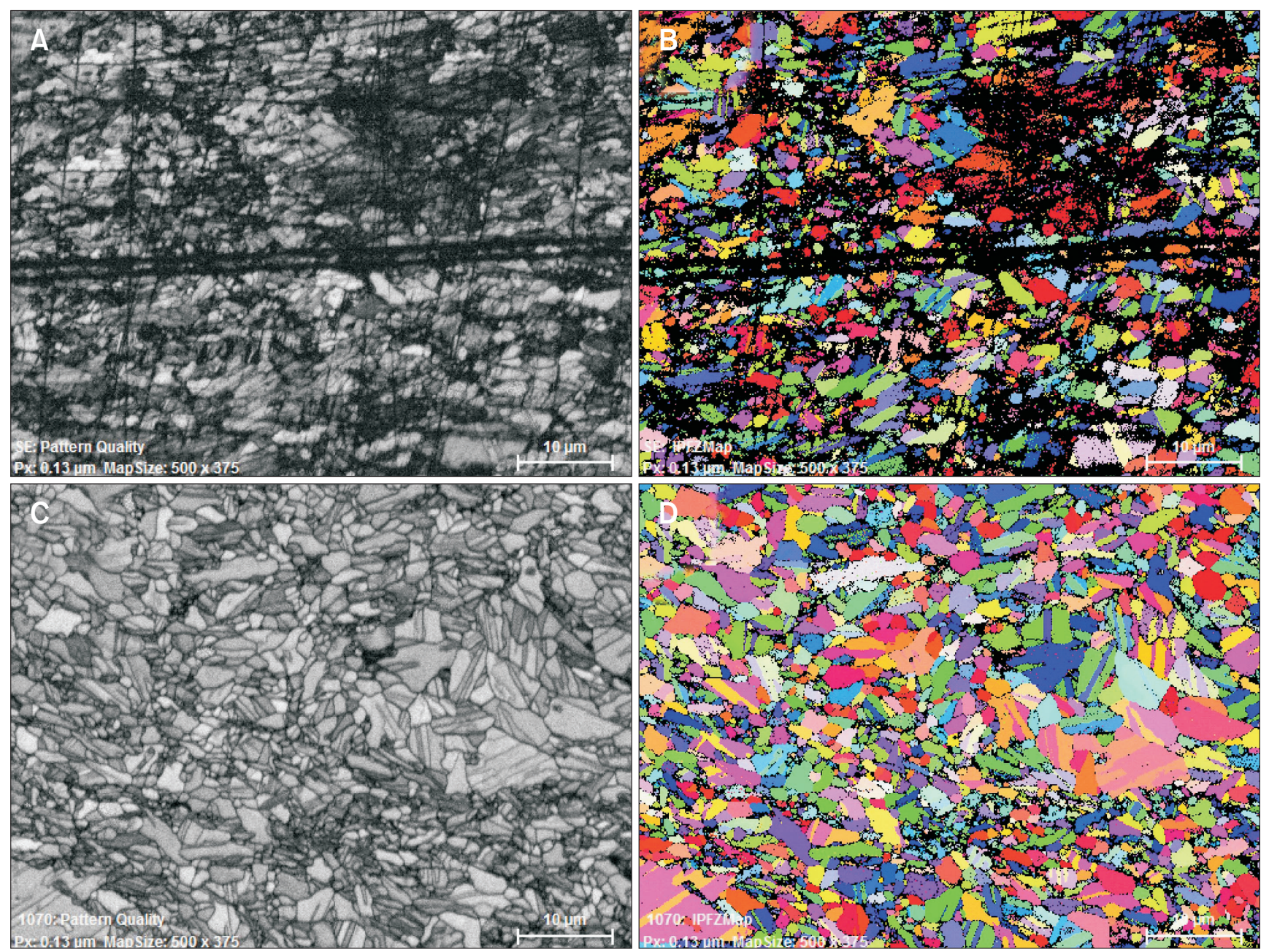

Fig. 5. Electron back scattered diffraction data acquired from $(\mathrm{Bi}, \mathrm{Sb})_{2} \mathrm{Te}_{3}$ samples prepared by colloidal silica polishing pattern quality map (A), normal direction orientation map (B), prepared by cross sectional ion miller pattern quality map (C), and normal direction orientation map (D). 
is continuously removed by the following incident ion beam. And the finally revealed sample surface remains free from these ion beam damage. So this cross sectional ion beam milling is applicable not only for the multilayered structure cross section observation (Kim et al., 2013) but also for the very soft materials such as $(\mathrm{Bi}, \mathrm{Sb})_{2} \mathrm{Te}_{3}$ which is deformable just by the moderate pressing by hand (Jung et al., 2015). Fig. 5 shows the comparison of EBSD data acquired by colloidal silica polishing (Fig. 5A and B) and cross sectional ion milling (Fig. 5C and D). As shown in Fig. $5 \mathrm{~A}$ and $\mathrm{B},(\mathrm{Bi}, \mathrm{Sb})_{2} \mathrm{Te}_{3}$ is too soft to make scratch free surface with mechanical polishing and it was almost impossible to get a reliable EBSD data. So the cross sectional ion milling was applied for the samples and we can get a reliable EBSD data enough to calculate grain boundary characteristics as shown in Fig. 5C and D.

\section{CONCLUSIONS}

1. EBSD sample preparation is the most important factor to determine whether we can get a reliable EBSD data or not.

2. Final polishing with reactive colloidal silica can help providing good EBSD patterns in most materials but the final polishing time should be limited.

3. Electro-chemical polishing is the one of the best sample preparation technique for EBSD in most metals and especially low temperature polishing $\left(-30^{\circ} \mathrm{C}\right)$ is recommended.

4. Ion beam milling can be applied for EBSD sample preparation in most materials but an ion beam damage during the milling should be considered to get the reliable EBSD data.

\section{CONFLICT OF INTEREST}

No potential conflict of interest relevant to this article was reported.

\section{REFERENCES}

Humphreys F J (1999) Determination of microtexture by EBSD at high spatial resolution in a FEGSEM. In: Proceedings of ICOTOM-12, ed. Szpunar JA, pp. 74-78, (NRC Research Press, Ottawa).

Jung S J, Park S Y, Kim B K, Kwon B, Kim S K, Park H H, Kim D I, Kim J Y, Hyun D B, Kim J S, and Baek S H (2015) Hardening of Bi-Te based alloys by dispersing B4C nanoparticles. Acta Mater. 97, 68-74.

Kim D I, Oh K H, Lee H C, Chang Y J, Sohn W S, and Jang J (2004) Characterization of crystallographic properties of SMC poly Si using electron backscattered diffraction. J. Microsc. 215, 121-126.

Kim D I, Paik J M, Joo Y C, Oh K H, Lee H C, and Dicks K (2002) Microtexture measurement of copper damascene line with EBSD. Mater. Sci. Forum 408-412, 529-534.

Kim J H, Kim D I, Shim J H, and Yi K W (2013) Investigation into the high temperature oxidation of Cu-bearing austenitic stainless steel using simultaneous electron backscatter diffraction-energy dispersive spectroscopy analysis. Corros. Sci. 77, 397-402.

Lassen N C K (1994) Automated Determination of Crystal Orientations from Electron Backscattering Patterns (Institut for Matematisk Modellering, Danmarks Tekniske Universitet, Lyngby).

Muppidi T, Field D P, Sanchez Jr J E, and Woo C (2005) Barrier layer, geometry and alloying effects on the microstructure and texture of electroplated copper thin films and damascene lines. Thin Solid Films 471, 63-70.

Schwartz A J, Kumar M, Adams B L, and Field D P (2010) Electron Backscatter Diffraction in Materials Science, 2nd Edition (Springer Science \& Business Media, New York).

Venables J A and Harland C J (1973) Electron back-scattering patterns: a new technique for obtaining crystallographic information in the scanning electron microscope. Phil. Mag. 27, 1193-1200. 